\title{
Contrasting predictive and causal values of predictors and of causes
}

\author{
OSKAR PINEÑO \\ State University of New York, Binghamton, New York \\ JAMES C. DENNISTON \\ State University of New York, Binghamton, New York \\ and Appalachian State University, Boone, North Carolina \\ TOM BECKERS \\ State University of New York, Binghamton, New York \\ and University of Leuven, Leuven, Belgium \\ HELENA MATUTE \\ University of Deusto, Bilbao, Spain \\ and \\ RALPH R. MILLER \\ State University of New York, Binghamton, New York
}

\begin{abstract}
Three experiments examined human processing of stimuli as predictors and causes. In Experiments $1 \mathrm{~A}$ and $1 \mathrm{~B}$, two serial events that both preceded a third were assessed as predictors and as causes of the third event. Instructions successfully provided scenarios in which one of the serial (target) stimuli was viewed as a strong predictor but as a weak cause of the third event. In Experiment 2, participants' preexperimental knowledge was drawn upon in such a way that two simultaneous antecedent events were processed as predictors or causes, which strongly influenced the occurrence of overshadowing between the antecedent events. Although a tendency toward overshadowing was found between predictors, reliable overshadowing was observed only between causes, and then only when the test question was causal. Together with other evidence in the human learning literature, the present results suggest that predictive and causal learning obey similar laws, but there is a greater susceptibility to cue competition in causal than predictive attribution.
\end{abstract}

This paper examines differences between predictive and causal learning in humans. Events often occur in our environment according to a consistent temporal distribution. Some events occur simultaneously (e.g., the sound and sight of water running out of the tap), whereas other events occur sequentially (e.g., hunger dissipates after the intake of food). When the events repeatedly take place following a sequential distribution in time, the first event (i.e., the antecedent event) can become a signal for the occurrence of the second event (i.e., the subsequent event). Learning to predict the occurrence of an event on

O.P. was supported by a postdoctoral fellowship from the Spanish Ministry of Education (Ref. EX2002-0739). H.M. was supported by Grant PI-2000-12 from the Department of Education, Universities, and Research of the Basque Government. T.B. was supported by a postdoctoral fellow by the Fund for Scientific Research Flanders (Belgium). We thank Jeffrey C. Amundson, Gonzalo P. Urcelay, Kouji Urushihara, Miguel A. Vadillo, and Daniel S. Wheeler for their comments on an earlier version of this manuscript. Correspondence should be addressed to R. R. Miller, Department of Psychology, SUNY-Binghamton, Binghamton, NY 13902-6000 (e-mail: rmiller@binghamton.edu). the basis of the occurrence of another event is essential for survival. For instance, we can learn to anticipate when and where food or water will be available, as well as which situations can potentially result in physical injuries or social reward or punishment. By learning to anticipate the occurrence of significant events, we can modify our behavior to maximize or minimize the consequences of appetitive or aversive events, respectively. But, under some conditions, we can learn that the antecedent event is not only a predictor, but also a cause of the subsequent event. Causal learning can be even more important than predictive learning. First, a cause is always a predictor of the event, so that causal learning might be expected to include and perhaps depend on predictive learning. Second, by knowing the causes of the event, we can not only anticipate the occurrence of the event, but also potentially control its occurrence. For example, we can learn to establish a comfortable temperature in a room by manipulating the thermostat, or we can learn that ingestion of a certain food produces an allergic reaction, in which case we will try to avoid that food in the future. Therefore, whereas predictive learning al- 
lows us to modify our behavior in order to adapt to the impending presence or absence of an event, causal learning also allows us to use our own behavior to produce or prevent the occurrence of an event.

The systematic study of causal learning by humans within an associative framework began two decades ago with a study by Dickinson, Shanks, and Evenden (1984). They reported blocking, an effect first found in classical conditioning with nonhuman animals (Kamin, 1968). Conventionally, in a blocking design, a conditioned stimulus (CS), A, is first paired with an unconditioned stimulus (US) and then CS A is further paired with the US in the presence of an added CS, X. At test, CS X elicits a weak response, relative to a control condition in which CS A was not paired with the US prior to the AX-US pairings. In the experiments by Dickinson et al., no actual CSs or USs were presented. Rather, the participants were exposed to neutral stimuli in a computer-based task resembling a video game and were instructed to rate the causal efficacy of an artillery shell being fired (analogous to a blocked CS X) in destroying a tank (analogous to a US). Additionally, a mine exploding (analogous to blocking a CS A) could also destroy the tank.

The relevance of the Dickinson et al. (1984) study to the area of human associative learning was twofold. First, as previously mentioned, these authors replicated the blocking effect with humans in a preparation in which (1) the fictitious stimuli were affectively neutral, and (2) the dependent variable used to assess the strength of the association between the candidate blocked cause, and the effect consisted of a verbal judgment (i.e., a numerical rating; see also Allan \& Jenkins, 1983; Alloy \& Abramson, 1979). Thus, this study showed that causal learning by humans, as in classical conditioning of nonhuman animals, is subject to the blocking effect. Second, this observation suggests that, regardless of the differences in species and preparations, the same mechanisms may be involved in conditioning and causal learning. As a logical consequence of this correspondence between conditioning and causal learning, Dickinson et al. (see also Gluck \& Bower, 1988; Shanks \& Dickinson, 1987) proposed that the associative theories developed to explain classical conditioning in nonhuman animals (e.g., Rescorla \& Wagner, 1972) could also be used to account for causal learning by humans.

Dickinson et al.'s (1984) study greatly stimulated the study of stimulus competition in humans within an associative framework. Stimulus competition effects, such as the above-mentioned blocking effect (Kamin, 1968), overshadowing (Pavlov, 1927), and relative stimulus validity effect (Wagner, Logan, Haberlandt, \& Price, 1968) were initially found in experiments using nonhuman animals and then successfully replicated using predictive or causal judgment preparations with humans (for a demonstration of overshadowing, see Waldmann, 2001; for demonstrations of relative stimulus validity effect, see Kao \& Wasserman, 1993; Matute, Arcediano, \& Miller, 1996; Van Hamme \& Wasserman, 1994). These studies of stimulus competition in human contingency learning demonstrated that, as in conditioning experiments with animals, responding to a target stimulus does not depend only on the number and quality of pairings with the outcome or US (i.e., contiguity), but also on the associative status of any other stimuli that were present during training of the target stimulus. According to different theoretical approaches, stimuli compete either to enter into an association with the outcome (e.g., Rescorla \& Wagner, 1972) or to express their previously acquired association with the outcome (e.g., Miller \& Matzel, 1988). Thus, by replicating effects previously found in nonhuman animals, the study of stimulus competition became a critical issue in the human associative learning literature. However, transfer between the animal conditioning and human associative learning literatures has not been unidirectional (see Miller \& Matute, 1996, for a detailed discussion). Some effects originally found in humans were also replicated in classical conditioning with nonhuman animals. This is the case for backward blocking, an effect akin to forward blocking but with the order of the training phases reversed. That is, AX-US pairings are followed by A-US pairings, and then responding to $\mathrm{X}$ is assessed. This effect was originally reported by Shanks (1985) in humans and then replicated in rats by Denniston, Miller, and Matute (1996).

The study of stimulus competition has attracted a large number of researchers and theorists in the area of human contingency learning, especially over the last two decades (see De Houwer \& Beckers, 2002, for a recent review). Two main paradigms have been commonly used indiscriminately in these studies: predictive and causal learning. In the predictive learning paradigm, participants are asked to rate the predictive relationship between a target antecedent event, normally referred to as cue or signal (or even predictor or indicator), and a subsequent event, referred to as the outcome $(\mathrm{O})$. In the causal learning paradigm, participants are asked to rate the causal relationship between the antecedent and subsequent events, which are normally referred to as cause and effect, respectively. Evidence of stimulus competition, mostly forward and backward blocking, has been reported in both the predictive and causal learning paradigms.

Although the study of predictive and causal learning in humans has received extensive attention, researchers have largely ignored possible differences between these two kinds of learning. Certainly there were almost no systematic comparisons of these two learning paradigms. The only exception to this was provided by De Houwer, Beckers, and Glautier (2002), who studied predictive and causal learning in both forward and backward blocking paradigms. In the causal conditions of their experiments (i.e., Condition Weapons), the participants were asked to rate the likelihood that firing a weapon would be followed by destruction of a tank (i.e., the outcome), whereas in the predictive conditions (i.e., Condition Indicators), they were asked to rate the likelihood of the destruction of the tank when an abstract visual figure 
was present. De Houwer et al. found both forward and backward blocking in the causal condition, but no stimulus competition in the predictive condition, which they interpreted as suggesting that stimulus competition depends on the causal nature of the stimuli.

Other studies have systematically manipulated the type of test questions (i.e., predictive or causal) in experiments in which the task is consistently embedded in what is clearly a causal scenario. For example, Matute, Vegas, and De Marez (2002; see also Vadillo, Miller, \& Matute, 2005) used a variation of the allergy task (Gluck \& Bower, 1988; Wasserman, 1990), in which the task of the participants was to rate the relationship between the ingestion of some substances (foods or medicines) and the development of fictitious allergies. Therefore, Matute et al. (2002) did not directly contrast predictors and causes per se, but rather the effect of different test questions concerning a potential cause. That is, in contrast to De Houwer et al. (2002), who contrasted predictors and causes but did not manipulate the type of test question, Matute et al. (2002) exclusively focused on the study of causal learning and manipulated the type of test question. Because these prior studies assessed the impact of either the type of stimuli (De Houwer et al., 2002) or the type of test question (Matute et al., 2002), an integrative assessment of the interaction of the type of stimuli (i.e., predictors vs. causes) and type of test question (i.e., predictive vs. causal) in a single study seemed necessary. Note that there are several different types of predictive questions that can be asked (see Vadillo et al., 2005). For brevity and because we used only one type of predictive question, we will refer to it simply as the predictive question, although it is highly similar to the predictive-value question of Vadillo et al. (2005) as opposed to what they called their prediction question.

In order to compare predictive and causal learning in a single experiment, at least three approaches could be followed. The first approach consists of using different scenarios (provided by the instructions) in the predictive and causal conditions. For example, the stock market task used by Chapman and Robbins (1990; see also Chapman, 1991), in which the participants are required to predict the behavior of the real estate market on the basis of changes in the price of stock for a fictitious electronics company, could be used in the predictive condition, whereas the allergy task (Wasserman, 1990) could be used in the causal condition. The problem with this approach is that it relies upon comparisons among groups differing appreciably in procedural aspects (e.g., instructions, set of stimuli, test questions, and rating scales). A second approach consists of using different instructions with the same scenario to encourage the processing of the relations as either predictive or causal, an approach that was followed by De Houwer et al. (2002). The third strategy consists of taking advantage of the participant's preexperimental knowledge of predictive and causal relations. For example, in the allergy task, participants are expected to process the intake of a medicine as a cause of the al- lergic reaction, whereas the color of a flask containing a medicine would be processed as a potential predictor.

Our present experiments used either different wording of instructions (Experiments 1A and 1B) or participants' preexperimental knowledge (Experiment 2), in order to study and contrast predictive and causal learning. Specifically, in Experiments 1A and 1B, the scenario had two antecedent events occur serially in time and then followed by the outcome (i.e., Stimulus 1 [S1] was followed by Stimulus 2 [S2], and S2 was followed by O). However, the instructions were worded so that both $\mathrm{S} 2$ and $\mathrm{O}$ were seemingly effects of S1. The purpose of Experiment 1 was simply to demonstrate that a single stimulus (S2 in this case) could simultaneously assume different values as a predictor and as a cause of O. In Experiment 2 , we used the training scenario to make two antecedent events either predictive or causal and then studied overshadowing between these events. The central issue in Experiment 2 was whether predictors and causes were differentially sensitive to overshadowing. The participants' preexperimental knowledge of the causal structure of the events was used to determine whether the training scenario was predictive or causal.

\section{EXPERIMENTS 1A AND 1B}

Experiments 1A and 1B examined the influence of the cover story provided within the instructions on the processing of stimuli as either predictors or causes of a subsequent stimulus. Although the antecedent stimuli (S1 and S2) and the subsequent stimulus (O) used in these experiments were simultaneously presented on the computer screen during each treatment trial, the instructions clearly indicated that the stimuli occurred serially. Specifically, according to the instructions, S1 was followed by S2, and then S2 was followed by O (see the top panel of Figure 1). In addition to the temporal priority of $\mathrm{S} 1$ over $\mathrm{S} 2$, the instructions encouraged processing of $\mathrm{S} 1$ as a common cause of both $\mathrm{S} 2$ and $\mathrm{O}$ (i.e., the presentation of S1 first S2 and then O; see bottom panel of Figure 1). On the basis of these causal relations suggested by the instructions, we anticipated the S1 would be perceived as both a predictor and a cause of $\mathrm{O}$. But how would S2 be perceived with respect to O? Cohen, Rundell, Spellman, and Cashon (1999) demonstrated that, in causal-chain sequences (i.e., $\mathrm{S} 1 \rightarrow \mathrm{S} 2 \rightarrow \mathrm{O}$ ), the first causal agent in the sequence (i.e., S1) is usually regarded as the only cause of $\mathrm{O}$. Although in Experiments $1 \mathrm{~A}$ and 1B the instructions were not designed to suggest a causal chain (i.e., in our experiments, the instructions suggested independent $\mathrm{S} 1 \rightarrow \mathrm{S} 2$ and $\mathrm{S} 1 \rightarrow \mathrm{O}$ causal relations, instead of a single $\mathrm{S} 1 \rightarrow \mathrm{S} 2 \rightarrow \mathrm{O}$ causal chain), on the basis of their findings, we expected (1) high predictive and causal ratings for $\mathrm{S} 1$ with respect to $\mathrm{O}$, and (2) high predictive ratings and low causal ratings for $\mathrm{S} 2$ with respect to $\mathrm{O}$. Although this result would be unsurprising, it would provide, if observed, a clear demonstration of the 
Temporal distribution of stimuli according to the instructions

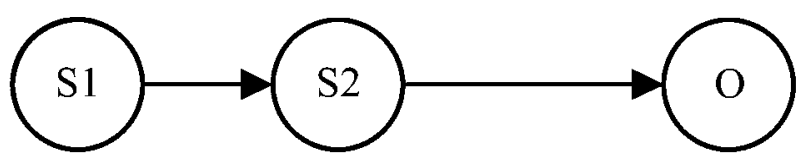

Causal relations provided by the instructions

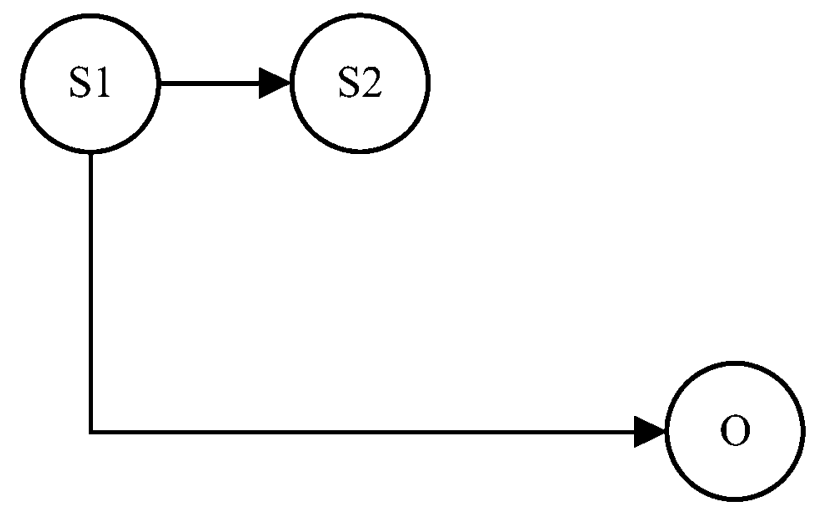

Figure 1. Experiments 1A and 1B. Top panel: temporal distribution of the stimuli, according to the instructions. Bottom panel: causal relations provided by the instructions. Time flows from left to right.

impact of a cover story in producing divergent predictive and causal processing of a single stimulus.

\section{Method}

Participants and Apparatus. Twenty-two undergraduate students from the State University of New York at Binghamton participated in Experiment 1A and 16 participated in Experiment 1B. The participants received either credits in partial fulfillment of a course requirement or $\$ 5$ for participating in these experiments. The experiments were conducted using personal computers in individual cubicles.

Procedure. The instructions for each scenario are provided in the Appendix. At the beginning of the experiment, all the participants were reminded of the difference between a predictor and a cause (see first screen of instructions in the Appendix). Four different scenarios were used in Experiments 1A and 1B. All participants were exposed to all four scenarios, counterbalanced for order. ${ }^{1}$ In Scenario 1, S1 consisted of "the gun being fired," S2 consisted of "the shell being ejected," and O was "the target being hit." In Scenario 2, S1 consisted of "imperfect steel sheets," S2 was "the alarm being sounded," and O was "defective bumpers being produced." In Scenario 3, S1 consisted of "the switch being turned," S2 consisted of "the floor being wet," and O was "the flowers blooming." In Scenario 4, S1 consisted of "workers loading strawberries," S2 consisted of "red stained shoes," and O was "workers wheezing." In each of these text-based scenarios, the participants were requested to read the instructions, pay attention to the training trials, and rate the predictive and causal value of the S1-O and S2-O relations at the end of training for that scenario. For each scenario, the participants were given 20 training trials in which S1, S2, and O could be either present or absent, with the restriction that no presentation of S1 occurred without the presentation of S2, and vice versa. Thus, the contingencies of $\mathrm{O}$ with respect to $\mathrm{S} 1$ and $\mathrm{S} 2$ were identical. Following training and testing with each of the first three scenarios, the participants were instructed: "You will now be learning about a new situation. What you previously learned is unrelated to this new situation. Please press the enter key to begin."

In Experiment 1A, $14 \mathrm{~S} 1-\mathrm{S} 2-\mathrm{O}$ trials, $0 \mathrm{~S} 1-\mathrm{S} 2-\mathrm{noO}$ trials, 2 noS1-noS2-O trials, and 4 noS1-noS2-noO trials were presented during training. This resulted in a contingency (i.e., as calculated by the unconditional $\Delta P$, Allan, 1980) of .67 for both the S1-O and S2$\mathrm{O}$ relations. In Experiment $1 \mathrm{~B}$, the same number of each trial type as in Experiment $1 \mathrm{~A}$ was administered during training in two of the four scenarios (i.e., high contingency). In the other two scenarios (i.e., zero contingency), the participants received $8 \mathrm{~S} 1-\mathrm{S} 2-\mathrm{O}$ trials, 2 S1-S2-noO trials, 8 noS1-noS2-O trials, and 2 noS1-noS2-noO trials, resulting in a contingency of .00 for both the S1-O and S2-O relations. The two types of contingencies were presented in counterbalanced order and also were counterbalanced across the different cover stories. The use of two contingencies allowed us to determine whether participants were sensitive to the training contingencies or merely responding on the basis of the instructions. The different trial types were pseudorandomly distributed across training, with the restriction that the first trial was always an S1-S2-O trial, and that no more than three trials of the same type occurred consecutively. A 1sec pause was introduced between trials, during which the computer screen remained blank. On each trial, the stimuli $(\mathrm{S} 1, \mathrm{~S} 2, \mathrm{O})$ were presented simultaneously in a horizontal sequence on the screen.

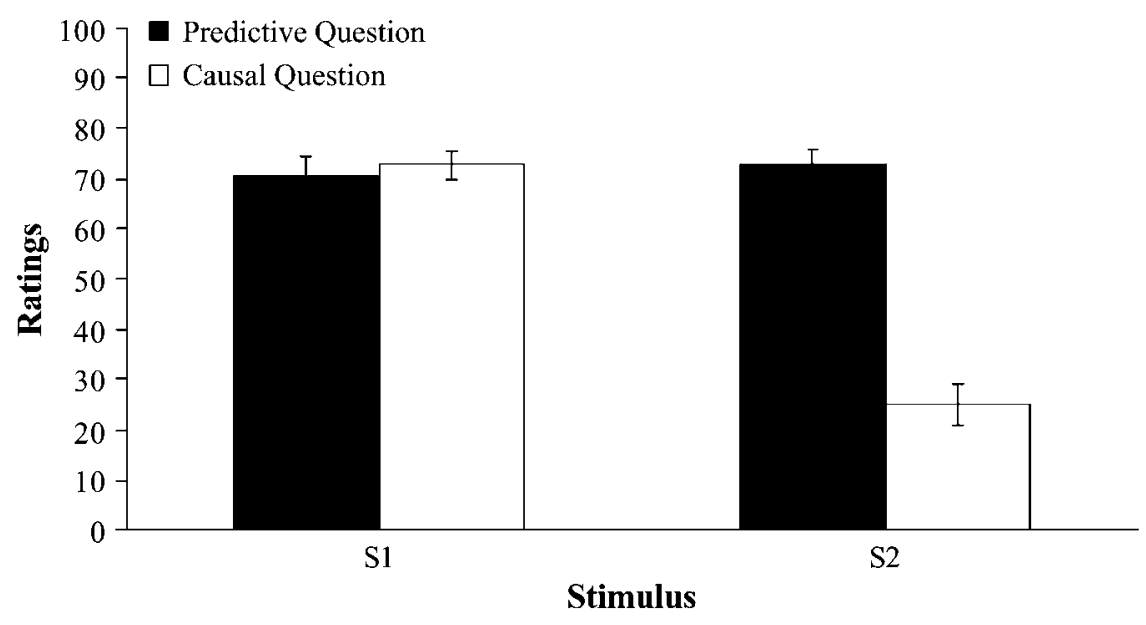

Figure 2. Results of Experiment 1A. A fully within-subjects design was used in this experiment. See text for details. 
Each stimulus was followed by a question mark and the presence or absence of each stimulus was denoted by the presentation of "yes" or "no" below the corresponding stimulus. Participants were able to advance to the next trial at their own pace by pressing the space bar, but only after the stimuli had been presented for at least 2 sec.

At the end of the training phase of each scenario, the participants were asked to rate the predictive and causal relationships between $\mathrm{S} 1$ and $\mathrm{O}$, and between S2 and O. Importantly, the predictive and causal questions differed only in the words predictor and cause, presented in uppercase. The predictive/causal test questions were as follows: "To what degree do you think that [S1 or S2] was a [PREDICTOR or CAUSE] of [O]?" The four test questions were presented together on the computer screen, counterbalanced for vertical order across participants. The participants could read all four of the questions before answering any of them, as well as answer them in any order. A rating scale was displayed below each test question. This scale ranged from 0 (not at all) to 100 (very high). An alpha level of $p<.05$ was adopted for all statistical analyses.

\section{Results and Discussion}

A preliminary 4 (scenario) $\times 2$ (stimulus: S1 vs. S2) $\times 2$ (test question: predictive vs. causal) analysis of variance (ANOVA) on the test ratings of Experiment 1A showed no main effect of scenario, nor any interaction involving scenario (all $p \mathrm{~s}>.19$ ). Therefore, the results of Experiment 1A were pooled across scenarios for each participant. These results are depicted in Figure 2. As can be seen, S1 was highly rated, as expected, as both a predictor and a cause of $\mathrm{O}$. S2 was given a low rating as a cause of $\mathrm{O}$; however, the predictive ratings of $\mathrm{S} 2$ were higher than its causal ratings and similar to the predictive ratings of S1. These impressions were confirmed by a 2 (stimulus: S1 vs. S2) $\times 2$ (test question: predictive vs. causal) ANOVA on the test ratings pooled from all four scenarios, which yielded main effects of stimulus $\left[F(1,21)=41.05, M S_{\mathrm{e}}=276.80, p<.001\right]$ and test question $\left[F(1,21)=31.19, M S_{\mathrm{e}}=364.60, p<.001\right]$, as well as a stimulus $\times$ test question interaction $[F(1,21)=$ $\left.76.04, M S_{\mathrm{e}}=178.85, p<.001\right]$. The source of this interaction was further examined using the Tukey test, which showed that the predictive and causal ratings of S1 did not differ. However, the causal ratings of S2 were lower than its predictive ratings. Also, the predictive ratings of $\mathrm{S} 1$ and S2 did not differ, but the causal rating of S2 was

\section{Condition High}

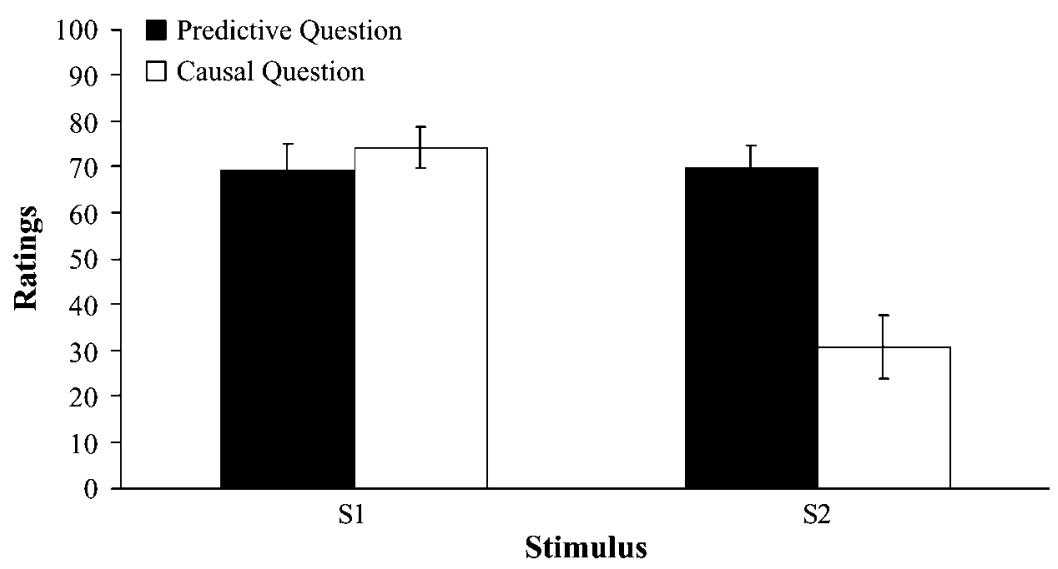

Condition Zero

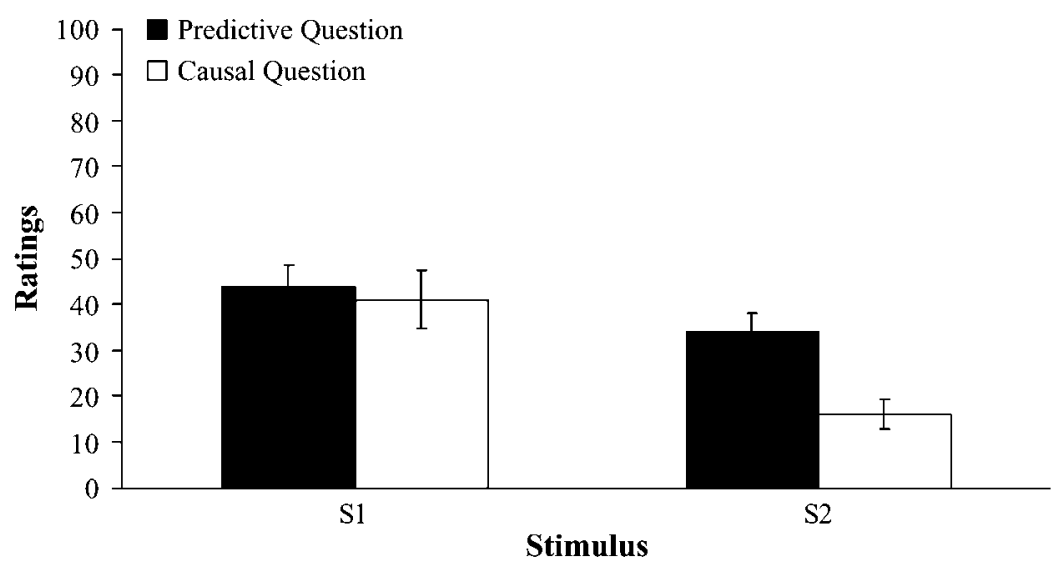

Figure 3. Results of Experiment 1B. The top and bottom panels depict the results of Conditions High and Zero, respectively. A fully within-subjects design was used in this experiment. See text for details. 
lower than that of S1. Thus, whereas $\mathrm{S} 1$ was rated as both a reliable predictor and a cause of $\mathrm{O}, \mathrm{S} 2$ was highly rated as a predictor, but not as a cause of $\mathrm{O}$.

In Experiment 1B, again there was no main effect or interaction with scenario; hence, data were pooled across scenarios for otherwise equivalent conditions. The results of Experiment 1B are depicted in Figure 3. As can be seen, the predictive and causal ratings of $S 1$ and $S 2$ in Condition High replicated those of Experiment 1A (i.e., high predictive and causal ratings of S1, and predictive ratings of S2 higher than its causal ratings). Also, the ratings were apparently sensitive to the contingencies. The ratings were lower in Condition Zero (bottom panel) than in Condition High (top panel). A pattern of results similar to that observed in Condition High was also found in Condition Zero. Most of these impressions were confirmed by a 2 (contingency: high vs. zero) $\times 2$ (stimulus: S1 vs. S2) $\times 2$ (test question: predictive vs. causal) ANOVA on the test ratings, which showed main effects of contingency $\left[F(1,15)=36.16, M S_{\mathrm{e}}=660.37, p<.001\right]$, stimulus $\left[F(1,15)=29.60, M S_{\mathrm{e}}=408.02, p<.001\right]$, and test question $\left[F(1,15)=17.28, M S_{\mathrm{e}}=344.90, p<.001\right]$. More important, this ANOVA also revealed a stimulus $\times$ test question interaction $\left[F(1,15)=22.90, M S_{\mathrm{e}}=\right.$ $306.79, p<.001]$ and a three-way interaction $[F(1,15)=$ $\left.5.45, M S_{\mathrm{e}}=304.78, p<.05\right]$. The remaining interactions were not significant $(p \mathrm{~s}>.15)$. Post hoc comparisons using the Tukey test on the data from Condition High revealed results similar to those of Experiment 1A. These comparisons showed that the predictive and causal ratings of S1 did not differ, and that the causal ratings of S2 were lower than its predictive ratings. Also, the predictive ratings of S1 and S2 did not differ, but the causal rating of S2 was lower than the causal rating of S1. In Condition Zero, post hoc comparisons using the Tukey test showed that, as in Condition High, the predictive and causal ratings of S1 did not differ. However, contrary to Condition High, in Condition Zero the causal and predictive ratings of S2 did not statistically differ, despite the tendency being in the same direction as in Condition High. Also, the causal ratings of S2 were lower than those of S1, whereas the predictive ratings of $\mathrm{S} 2$ and $\mathrm{S} 1$ did not differ.

Overall, these results show that, with a moderately high positive contingency (i.e., $\Delta P=.67$, Experiment $1 \mathrm{~A}$ and Condition High in Experiment 1B), S1 was highly rated as both a predictor and cause of Stimulus $\mathrm{O}$, whereas $\mathrm{S} 2$ was highly rated as a predictor, but not as a cause of O. In other words, the instructions concerning causal mechanisms, which encouraged the processing of S2 as a mere predictor and $\mathrm{S} 1$ as a cause of $\mathrm{O}$, yielded differential predictive and causal value of the stimuli despite their having identical contingencies with respect to $\mathrm{O}$.

Although the results of Experiments 1A and 1B suggest that the causal mechanisms implicit in the scenarios favored processing of stimuli as either a predictor or a cause, overshadowing of S2 by S1 based on the temporal priority of $\mathrm{S} 1$ could provide an alternative explanation of the observed results. That is, the serial presenta- tion of S1 and S2 engendered in the instructions could have allowed for these stimuli to compete with one another for the acquisition of the predictive and causal value. Because Experiments 1A and 1B did not include a control condition for overshadowing (i.e., a condition in which S2 was trained with the outcome in the absence of S1), these experiments do not permit distinguishing between causal mechanism and temporally based stimulus competition as the basis for $\mathrm{S} 2$ being rated as a strong predictor and a weak cause. Therefore, although the cover stories provided by the instructions in Experiments $1 \mathrm{~A}$ and $1 \mathrm{~B}$ seemingly produced differential processing of S2 as a predictor and as a cause, the role played in the present results by participants' preexperimental knowledge concerning predictive and causal relationships in the real world is unclear. Experiment 2 was designed to eliminate the serial relationship of the antecedent events, among other things.

\section{EXPERIMENT 2}

The results of Experiments 1A and 1B suggested that either temporal priority or the participants' preexperimental knowledge of the predictive and causal relations established by the fictitious scenarios favored processing of the target stimulus, S2, more as a predictor than as cause. Experiment 2 also assessed predictive and causal values of a stimulus, but this time we assessed overshadowing between simultaneous antecedent stimuli. Overshadowing was separately assessed between predictors (i.e., Group P) and between causes (i.e., Group C). Unlike Experiments 1A and 1B, in Experiment 2 the simultaneous occurrence of the two antecedent events precluded temporal order from possibly contributing to differentiation between predictors and causes. Instead, differentiation between predictors and causes was provided exclusively by the participants' preexperimental knowledge of predictive and causal relations in the real world. Thus, any differences in overshadowing observed between predictors and between causes would presumably be due to differences in the sensitivity of predictors and causes to associative competition.

In Experiment 2 the instructions contained no explicit information concerning the role of the stimuli as predictors or causes. All participants were given a common set of instructions that was neutral regarding the causal/ predictive nature of the task in order to minimize the influence of instructions on ratings as a function of their being predictive or causal. The participants' task was to take a fictitious test for a position in the National Weather Service. For all the participants, the outcome consisted of the occurrence of a thunderstorm. However, different antecedent stimuli were presented to Groups $P$ and C. In Group P the stimuli consisted of instances of animal behavior (e.g., birds flying in circles), whereas in Group $\mathrm{C}$ the stimuli consisted of variations in the climatic conditions (e.g., sudden change of temperature). Although both types of stimuli were clearly correlates of 
the subsequent occurrence of a thunderstorm, the stimuli were expected to be processed as mere predictors of the thunderstorm in Group P, and as causes of the thunderstorm in Group C.

In Experiments 1A and 1B, S1 (an implicit cause of O) might have overshadowed the causal value of S2 (the target stimulus), but there was no control condition to assess overshadowing. Experiment 2 investigated whether the causal value and predictive value of stimuli are subject to overshadowing by other causes and by other predictors, respectively, relative to appropriate overshadowing control conditions. In Experiment 2, participants received training with two implicit causes or two implicit predictors (i.e., Stimuli A and X) presented in compound, as well as a control condition for overshadowing (i.e., Stimulus Y). All participants were asked both predictive and causal test questions in order to ascertain whether overshadowing of the predictive or causal value of a predictor (i.e., Group P) or of a cause (i.e., Group C) could be observed. As in Experiments 1A and $1 \mathrm{~B}$, the predictive and causal test questions differed only in one word (i.e., predictor vs. cause).

\section{Method}

Participants and Apparatus. The participants were 74 students in an introductory psychology course at the State University of New York at Binghamton, who participated in this experiment in partial fulfillment of a course requirement. The participants were randomly assigned to one of two groups ( $n$ s $=37)$.

Procedure. The task used in Experiment 2 simulated a computerbased test that the participants had to take as part of a fictitious application for the National Weather Service. ${ }^{2}$ At the beginning of the experiment, all participants were presented with a screen of instructions (see the Appendix). Immediately after they had read the instructions, training commenced.

During training, the participants were given $5 \mathrm{AX}-\mathrm{O}$ and $5 \mathrm{Y}-\mathrm{O}$ trials, in pseudorandom order. In Group P, Stimuli A, X, and Y consisted of the presentation of the text "Birds flying in circles," "Dogs barking," and "Squirrels returning to nests," counterbalanced. In Group C, these stimuli consisted of the presentation of the text "Fall of atmospheric pressure," "Sudden change of temperature," and "High humidity," counterbalanced. The O consisted of the presentation of the text "Thunderstorm: Intensity $=10$ out of $20 . " 3$

After each trial and before the interval separating it from the subsequent trial, the participants were required to indicate whether the stimuli (i.e., A, X, Y, and O) were present or absent on that specific trial. In this screen, the following instructions were presented: "Please, indicate whether the following events were present or absent on this occasion." All of the stimuli were presented in a vertical list format on each trial, counterbalanced for order, together with a box labeled "This event was ...," containing the following two options: "Present" and "Absent." The participants were asked to use the computer mouse to indicate the presence or absence of each stimulus before they were allowed to proceed to the next trial; they received no feedback concerning their answers. The purpose of this posttrial test was twofold. First, it encouraged the participants to pay close attention to the events. Second, it provided a criterion to eliminate those participants who did not pay sufficient attention during training. In order for a participant's data to be retained in the analyses, she/he had to correctly indicate the presence or absence of all the stimuli on at least two trials among Trials $2-5$ of AX-O and two trials among Trials $2-5$ of Y-O (i.e., they were always allowed to fail in the first AX-O and Y-O trials because this first test was expected to increase attention to stimuli on sub- sequent trials). The application of this criterion eliminated 17 participants ( 8 from Group P and 9 from Group C).

During testing, the participants were presented with predictive and causal questions concerning the A-O, X-O, and Y-O associations. The predictive and causal questions read as follows: "To what degree do you think that [A or X or Y] is a [PREDICTOR or CAUSE] of thunderstorms?" All six of the test questions were presented on a single screen. The order of presentation of the test questions corresponding to a physical stimulus (e.g., dogs barking) was identical for all participants. However, because of the complete counterbalancing of the assignment of these text labels to the different stimuli (i.e., A, X, and Y), the order of presentation of the test questions of the stimuli was counterbalanced. Also, the predictive and causal test questions were presented in a counterbalanced order (i.e., all predictive first or all causal first). The participants were requested to answer each question by rating the different predictive and causal relations using a scale ranging from -10 to +10 . A brief note at the top of the screen explained the meaning of the extreme and middle values of the scale as well as how to indicate the rating: "In order to answer, use any number in the scale from -10 to +10 , where $-10=$ Not at all, $0=$ Not sure, and $+10=$ Very high. Click on the vertical arrows to indicate your rating. Please read ALL of the questions before you answer any of them." The current rating to each question could be viewed in a small window to the right of the corresponding question. This rating, which was initially set at 0 , could be changed by clicking on one of two arrows (up or down). Clicking on the up or down arrows yielded a \pm 1 increment or decrement, respectively, in the rating. Once the participants had rated the different predictive and causal relations, they had to click on a button labeled "Save my ratings" in order to proceed to the next screen.

Following testing, the participants were given a short quiz in order to assess their ability to differentiate prediction from causality. This quiz consisted of three multiple choice questions, which read as follows: "Question 1: Firing a gun is __ by the pulling of its trigger"; "Question 2: A train coming is__ by a flashing red light at a railroad crossing"; and "Question 3: High tides are by singing pop songs." For each question, the participants had to choose among the following three answers: "predicted," "caused," "neither predicted nor caused." The correct answers for Questions 1, 2, and 3 were "caused," "predicted," and "neither predicted nor caused," respectively. Participants who failed to answer all the questions correctly were removed from the analyses. Among the participants who passed the attentional criterion (i.e., correctly indicating the presence or absence of all the stimuli on at least two of each type of training trial), 5 participants (i.e., 2 and 3 participants from Groups P and C, respectively) failed to pass the present criterion. Thus, after the participants that did not pass both criteria were eliminated, the resulting sample of participants in each group was 27 for Group P and 25 for Group C.

Prior to analysis, the ratings of Stimuli $\mathrm{A}$ and $\mathrm{X}$ were averaged to obtain a single rating for stimuli trained in compound (i.e., Comp). Pooling the ratings of Stimuli $\mathrm{A}$ and $\mathrm{X}$ was justified on the basis of the complete counterbalancing of the stimuli and was performed in order to reduce variability of the ratings. The ratings of Stimulus Y directly represented the ratings of the elementally trained stimulus (i.e., Elem). Therefore, Stimuli Comp and Elem hereafter will represent the different training conditions received by Stimuli A and X (i.e., compound training) and Stimulus Y (i.e., elemental training).

\section{Results and Discussion}

The order of presentation of the predictive and causal questions at test was found to influence the test results. Thus, in order to minimize the influence of the order of presentation of the predictive and causal test questions on the test results, we performed the analyses exclusively 
on the ratings corresponding to the first test question asked concerning each stimulus. After the second ratings were eliminated, the sample for predictive ratings consisted of 14 participants for each of Groups $\mathrm{P}$ and $\mathrm{C}$ and the sample for causal ratings consisted of 13 participants for Group P and 11 participants for Group C.

Figure 4 depicts the results of Experiment 2. As can be seen, overshadowing (i.e., lower ratings of Stimulus Comp than of Stimulus Elem) was suggested in Group P (top panel) with the predictive ratings and in Group C (bottom panel) with both the predictive and the causal ratings. Also, overshadowing was apparently stronger in Group C (as assessed by the causal ratings) than in Group P (as assessed by the predictive ratings). These impressions were partially supported by a 2 (type of stimuli: P vs. C) $\times 2$ (test question: predictive vs. causal) $\times 2$ (training condition: Comp vs. Elem) ANOVA on the judgmental rat- ings. This ANOVA yielded main effects of type of stimuli $\left[F(1,48)=5.40, M S_{\mathrm{e}}=32.71, p<.05\right]$, test question $\left[F(1,48)=34.10, M S_{\mathrm{e}}=32.71, p<.01\right]$, and training condition $\left[F(1,48)=20.79, M S_{\mathrm{e}}=5.55, p<.01\right]$. More importantly, there were significant interactions: type of stimuli $\times$ test question $\left[F(1,48)=4.62, M S_{\mathrm{e}}=32.71\right.$, $p<.05]$, type of stimuli $\times$ training condition $[F(1,48)=$ $\left.17.43, M S_{\mathrm{e}}=5.55, p<.01\right]$, and a three-way interaction $\left[F(1,48)=18.41, M S_{\mathrm{e}}=5.55, p<.01\right]$. Post hoc comparisons using the Tukey test indicated overshadowing (i.e., lower ratings of Comp than Elem) in Group C, as assessed by the causal ratings. Despite the tendency toward overshadowing in the predictive ratings in both Groups $\mathrm{P}$ and $\mathrm{C}$ suggested by Figure 4, post hoc comparisons showed no significant difference between the predictive ratings of Comp and Elem in either Group $\mathrm{P}$ or Group C. It is a little difficult to appreciate the criti-
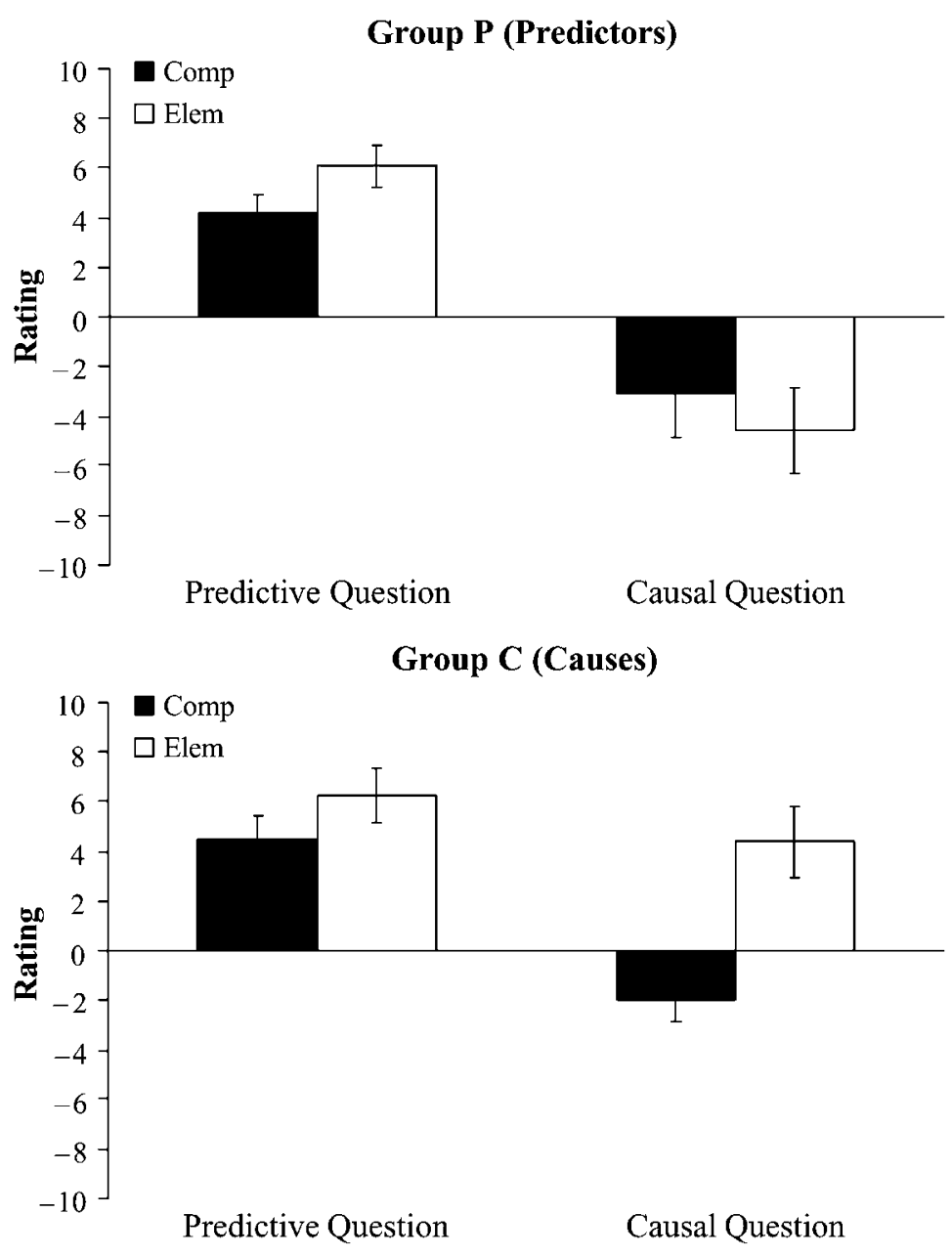

Figure 4. Results of Experiment 2. The top and bottom panels depict the mean ratings in Groups $P$ (predictors) and $C$ (causes), respectively. For Group P, participants' preexperimental knowledge was expected to encourage purely predictive processing of the relation between the antecedent and subsequent paired events. For Group C, it was expected to encourage causal processing of the relation between the antecedent and subsequent paired events. See text for details. 
cal differences in Figure 4. Consequently, we converted the ratings of Stimuli Comp and Elem into overshadowing scores (i.e., Overshadowing Scores = Rating Elem - Rating Comp). Figure 5 depicts these overshadowing scores for the predictive and causal test questions in Groups P and C. Consistent with the previous statistical analysis, overshadowing is seen to be stronger in the causal ratings of Group $\mathrm{C}$ than in the predictive ratings of both Groups $\mathrm{C}$ and $\mathrm{P}$.

\section{GENERAL DISCUSSION}

The present series of experiments used two techniques to assess the processing of stimuli as predictors or causes. In Experiments 1A and 1B, two stimuli (i.e., S1 and S2) identically covaried with the presentation of a third stimulus (i.e., O). However, the instructions used in these experiments promoted the processing of $\mathrm{S} 1$ as a cause of both $\mathrm{O}$ and S2 (see Figure 1). The results of these experiments showed, perhaps unsurprisingly but now in a controlled manner, that although S2 was rated as a poor cause of $\mathrm{O}$, its predictive value was high. However, Experiments $1 \mathrm{~A}$ and $1 \mathrm{~B}$ raised the question of whether the impaired causal value of S2 was exclusively due to the instructions having created a bias against the processing of $\mathrm{S} 2$ as a cause of O or, instead, Stimulus S1 having overshadowed $\mathrm{S} 2$ as a cause but not as a predictor, or a combination of these two factors.

Experiment 2 assessed whether overshadowing could occur in a similar preparation, but one in which the instructions did not identify one antecedent event as a cause and the other as a predictor. Instead, the role of the stimuli as predictors or causes was manipulated using participants' preexperimental knowledge and, orthogonally, both the predictive and the causal value of the stimuli were assessed during testing. This experiment used a single scenario and a common $\mathrm{O}$ for both the predictive and causal conditions, and manipulated the predictive and causal relations by varying the kind of antecedent stimuli presented in each condition. The participants' preexperimental knowledge concerning the different stimulus-O relations as either predictive or causal made the scenario predictive or causal, respectively. This approach allowed us to discount alternative explanations of differences in processing of predictors and causes based on the wording of the instructions (e.g., De Houwer et al., 2002). Consistent with many other reports in the literature (e.g., Waldmann, 2001), Experiment 2 showed overshadowing between causes (i.e., Group C) as assessed by the causal test question, but not as assessed by the predictive test question. Moreover, overshadowing was not found between predictors (i.e., Group P), as assessed by either the predictive or causal test questions. However, a nonsignificant tendency toward overshadowing was observed in the predictive ratings of predictors and causes. Analogously, a nonsignificant difference in the opposite direction was found in the causal ratings of predictors. But we hesitate to even speculate concerning what this might suggest because the meaning of negative causal ratings is unclear in our preparation.

The present results were observed despite our using nearly identical wording of the predictive and causal questions in Experiments 1A and 1B, as well as in Experiment 2 , in order to avoid unnecessary differences between test questions. Because of this, the predictive question might have been expected to resemble the causal question in many aspects, such as for example its stimulating a comparison between the predictive value of the target stimulus and any other candidate predictor, thereby heightening its sensitivity to the impact of the predictive value of other stimuli (i.e., stimulus competition). In summary, the results of Experiment 2 suggest that responding based on causal learning is a more selective process than is responding based on predictive learning. This higher sensitivity of causes to competition does not imply that predictors do not compete. Although Experiment 2 revealed no significant overshadowing between predictors (i.e., Group P, predictive ratings), a tendency

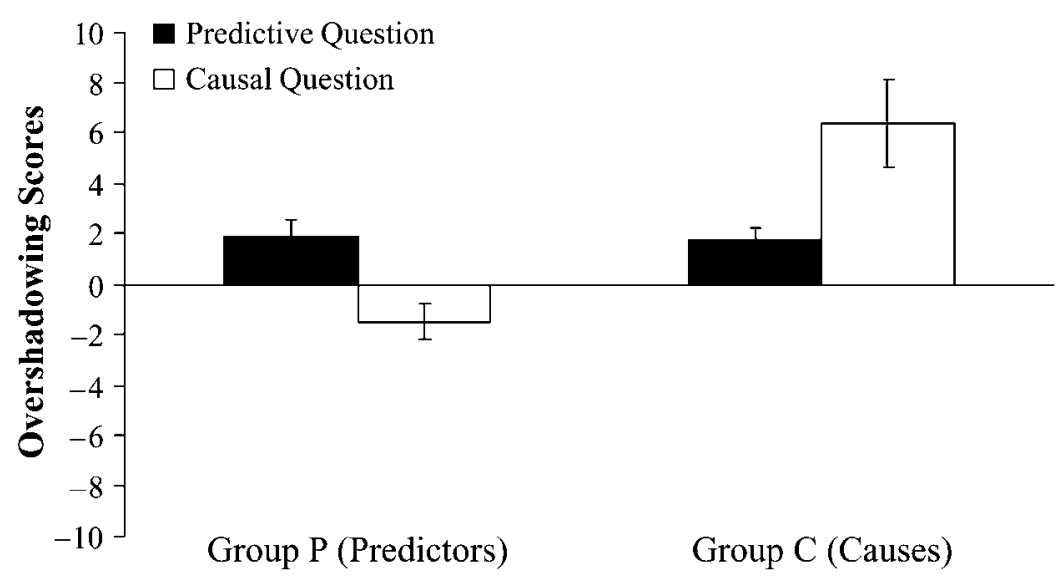

Figure 5. Results of Experiment 2. Mean ratings converted into overshadowing scores (i.e., overshadowing scores $=$ rating of element - rating of compound). See text for details. 
toward overshadowing was found. Also, it is important to remember that stimulus competition effects have been reported in the literature, not only among causes, but also among predictors. This higher sensitivity of causal learning compared with predictive learning with respect to stimulus competition cannot be explained by any contemporary model in its current form. According to associative models of learning (e.g., Dickinson \& Burke, 1996; Mackintosh, 1975; Miller \& Matzel, 1988; Pearce \& Hall, 1980; Rescorla \& Wagner, 1972; Van Hamme \& Wasserman, 1994; Wagner, 1981), learning consists of the formation and strengthening of associations between the representations of antecedent and subsequent events. These models do not differentiate between processing of these relationships as cue-O (predictive learning) or cause-effect (causal learning) relationships; therefore, they are silent concerning any difference between predictive and causal learning.

The present results also pose problems for statistical models of human learning. Most of these models (e.g., Allan's, 1980, unconditional $\Delta P$; Cheng \& Novick's, 1992 , focal-set theory) do not try to differentially account for predictive and causal learning; rather, they are designed to explain covariation learning (i.e., learning whether the occurrence of one event covaries with the occurrence of another event) without differentiating it from either prediction or causation. Other models that have been explicitly developed in order to explain causal learning in humans (e.g., Cheng, 1997; Spellman, 1996) are also silent concerning predictive learning. According to these models, learning the covariation (i.e., as measured by the unconditional $\Delta P$ ) among the stimuli is a necessary condition in order to learn different causal relations. Causation results exclusively from the history of training with certain covarying events and from controlling for alternative causes of the outcome. Controlling for the alternative causes takes the form of adjusting $\Delta P$ relative to the base-rate occurrence of the outcome [i.e., $1-P(\mathrm{O} \mid$ noC $)]$ in Cheng's Power-PC model, and of the computation of a conditional contingency (i.e., the contingency of the target candidate cause relative to the contingency of the alternative candidate cause or causes) in Spellman's model. Importantly, predictive learning is not equated with covariation learning in these models. Thus, these models are also silent on the difference between predictive and causal learning.

Waldmann's $(1996,2000,2001)$ causal-model theory explicitly differentiates between causes and effects, but not between predictors and causes. In Waldmann's causal-model view, the participants' intrinsic knowledge concerning causal relationships (i.e., causes influence effects, but not vice versa) results in competition between causes, but not between effects. This directionality, independent of the order in which the events are presented, is the basis of the causal model. Although published versions of Waldmann's model do not address the nature of predictors, it could explain the present results by assuming that, in contrast to causes (which are necessarily antecedent events for the outcome), predictors are effects that can be used to diagnose a cause. This possibility can be applied to our Experiment 2, in which several examples of animal behavior were used as predictors of a thunderstorm. If participants inferred that these patterns of animal behavior were indeed elicited by atmospheric conditions that preceded the thunderstorm (e.g., dogs barked in response to the impending thunderstorm), then no overshadowing should be observed in the predictive condition because, according to Waldmann's causal model, only causes (but not effects), compete.

In sum, most current models of human learning cannot explain the observed greater stimulus competition between causes than between predictors (but see De Houwer et al., 2002, for an account based on inferential reasoning). However, some current models could be adapted post hoc to explain these results. For example, statistical models (e.g., Cheng, 1997; Cheng \& Novick, 1992 ) could be adapted by using a weighted $\Delta P$ (see, e.g., Kao \& Wasserman, 1993), in which each cell in the traditional $2 \times 2$ contingency matrix is multiplied by a given value ranging from 0 to 1 , and making the weights for the cells $c$ (i.e., cue absent and outcome present) and $d$ (i.e., both cue and outcome absent) higher for causal learning than for predictive learning. If the trials during which the target cue is absent are more heavily weighted for causal judgments than for predictive judgments, stimulus competition would be stronger in the former than in the latter case. Although this adaptation of $\Delta P$ could explain stronger blocking in causality than in predictive value, it still would face problems in explaining stronger overshadowing in causality than in predictive value because in a between-subjects overshadowing design there are no trials on which the target cue is absent. However, this is the case only with a between-groups methodology; with a within-subjects design such as the one we used in Experiment 2, the participants receive both $\mathrm{AX}-\mathrm{O}$ and $\mathrm{Y}-\mathrm{O}$ trials, and the $\mathrm{Y}-\mathrm{O}$ trials constitute $\mathrm{O}$ trials without $\mathrm{X}$ present.

Another model that could be adapted to account for these results is the comparator hypothesis (Miller \& Matzel, 1988). According to this theory, overshadowing and other stimulus competition effects consist of impaired responding elicited by the target stimulus due to its previous training in compound with a competing stimulus. Specifically, this theory assumes that the presentation of the target stimulus, $\mathrm{X}$, directly activates the representation of $\mathrm{O}$ (i.e., through the $\mathrm{X}-\mathrm{O}$ association), as well as the representation of the competing stimulus, A (i.e., through an X-A within-compound association), which in turn indirectly activates the representation of $\mathrm{O}$ (i.e., through an A-O association). According to the comparator hypothesis, responding to $\mathrm{X}$ is based on a comparison between the strength of the direct activation of $\mathrm{O}$ (i.e., proportional to the strength of the $\mathrm{X}-\mathrm{O}$ association) and the strength of the indirect activation of $\mathrm{O}$ (i.e., proportional to the product of the strengths of the $\mathrm{X}-\mathrm{A}$ and $\mathrm{A}-\mathrm{O}$ associations). The behavioral effect of the 
directly activated representation of the outcome is downmodulated by the indirectly activated representation of the outcome. Hence, a weak response to the target stimulus is observed in stimulus competition situations. In order to explain the results of the present experiments, this model could be adapted by assuming that the comparison process has greater impact on causal than on predictive responding. For example, if the value of the indirect activation of $\mathrm{O}$ is weighted so that it is larger in causal than in predictive learning (i.e., analogous to the case of cells $c$ and $d$ in the previous adaptation of $\Delta P$ ), then the interference produced by the competing association on the expression of the target association would be stronger in causal judgment than in predictive judgment. More important, this adaptation of the comparator hypothesis, as well as the adapted $\Delta P$, does not preclude the possibility of stimulus competition being observed between predictors.

It must be pointed out that this post hoc adaptation of both the $\Delta P$ model (e.g., Allan, 1980) and the comparator hypothesis (Miller \& Matzel, 1988) to explain the present results does not merely assume that causes are, per se, more susceptible to competition than are predictors. As indicated by the findings of Experiment 2, only the causal value of causes (i.e., as assessed by the causal test question) was strongly affected by a stimulus competition treatment (i.e., overshadowing in our experiment). This interaction between the nature of the stimuli (as predictors or causes) and the test question (as predictive or causal) suggests that causal responding, instead of causal learning, is highly susceptible to competition. This emphasis on responding rather than learning is consistent with our proposed modifications of these models. Obviously, because mere predictors lack a causal value, the weaker stimulus competition found between predictors than between causes can be explained by the intrinsic inability of predictors to support a causal response or rating.

Of course, the $\Delta P$ model and the comparator hypothesis are not the only models that could be extended to account for the higher susceptibility of causal judgments, relative to predictive judgments, to stimulus competition. Models are flexible and they can encompass different post hoc adaptations to explain the present results. For example, traditional associative models (e.g., Rescorla \& Wagner, 1972) could assume independent causal and predictive learning, with causal learning proceeding more rapidly than predictive learning (e.g., learning-rate parameters may have higher values in causal associative learning than in predictive associative learning). Applied to Experiment 2 , this principle anticipates greater overshadowing between causes than between predictors, at least after a few pairings of the AX compound with $\mathrm{O}$, which is what was observed. In this framework, predictive test questions assess predictive learning and causal questions assess causal learning.

In Experiment 2, overshadowing was observed only when the causal status of an antecedent event was as- sessed at test. Because participants received the predictive and causal test questions for the first time during the test phase, the test questions could not affect predictive or causal learning during the overshadowing treatment. Moreover, differences in competition between causes and between predictors have been observed for the same stimuli (e.g., Experiment 2), which means that the basis for the differential competition is present independently of the test question. This suggests (but certainly does not prove) that participants have different learning functions during training for predictive value and for causal value. However, even if this is correct, the actual competition could occur during training (i.e., an acquisition deficit) or during testing (i.e., a performance deficit).

The general assumption that causal learning is a more selective process regarding stimulus competition than is predictive learning, although speculative, is interesting. As humans, we need to both predict and control the events that occur in our environment. In both predictive and causal learning, selecting the most reliable antecedent stimulus (i.e., either predictor or cause) of an event can be of critical importance for adaptative purposes. However, causal learning might be more sensitive to stimulus selection effects than predictive learning because, in order to efficiently influence the occurrence and/or intensity of an event, we must exactly identify and then manipulate its cause and avoid response competition arising from manipulating other candidate causes. In contrast, attending to both unique and redundant predictors to anticipate outcomes seemingly would not be as injurious. Alternatively worded, it is likely more difficult to simultaneously manipulate many causes than it is to simultaneously attend to many predictors. This would result in selection among candidate causes being a more rigorous process than selection among candidate predictors. As previously stated, this does not mean that no stimulus competition will ever occur among predictors. In fact, Experiment 2 revealed a tendency to stimulus competition among predictors. It simply implies that stimulus competition would be stronger in causal judgment than in predictive judgment.

The evolutionary question is, thus, why has causal learning evolved as a more selective process than predictive learning? The answer to this question likely originates in predictors usually coming from the environment and causes often originating in our own instrumental behavior. More generally, learning about the relations between external events can be a form of either predictive or causal learning, whereas learning about the relations between our own responses and their consequences is causal by nature (see Dickinson, 2001). A corollary of this view is that competition among responses (e.g., response-outcome relations in instrumental conditioning) should be more readily observed than competition among stimuli. But such a comparison in a well-controlled situation has not yet been performed. The present study should be considered a first attempt to contrast two closely related types of learning, predictive and causal. 


\section{REFERENCES}

Allan, L. G. (1980). A note on measurement of contingency between two binary variables in judgment tasks. Bulletin of the Psychonomic Society, 15, 147-149.

Allan, L. G., \& Jenkins, H. M. (1983). The effect of representations of binary variables on judgment of influence. Learning \& Motivation, 14, 381-405.

ALLOY, L. B., \& ABRAmson, L. Y. (1979). Judgment of contingency in depressed and nondepressed students: Sadder but wiser? Journal of Experimental Psychology: General, 108, 441-485.

CHAPMAN, G. B. (1991). Trial order affects cue interaction in contingency judgment. Journal of Experimental Psychology: Learning, Memory, \& Cognition, 17, 837-854.

Chapman, G. B., \& RobBIns, S. J. (1990). Cue interaction in human contingency judgment. Memory \& Cognition, 18, 537-545.

Cheng, P. W. (1997). From covariation to causation: A causal power theory. Psychological Review, 104, 367-405.

Cheng, P. W., \& Novick, L. R. (1992). Covariation in natural causal induction. Psychological Review, 99, 365-382.

Cohen, L. B., Rundell, L. J., Spellman, B. A., \& Cashon, C. H. (1999). Infant's perception of causal chains. Psychological Science, 10, 412-418.

De Houwer, J., \& Beckers, T. (2002). A review of recent developments in research and theories on human contingency learning. Quarterly Journal of Experimental Psychology, 55B, 289-310.

De Houwer, J., Beckers, T., \& Glautier, S. (2002). Outcome and cue properties modulate blocking. Quarterly Journal of Experimental Psychology, 55A, 965-985.

Denniston, J. C., Miller, R. R., \& Matute, H. (1996). Biological significance as a determinant of cue competition. Psychological Science, 7, 325-331.

DiCKInSON, A. (2001). The Twenty-Eighth Bartlett Memorial Lecture. Causal learning: An associative analysis. Quarterly Journal of Experimental Psychology, 54B, 3-25.

DiCKINSON, A., \& BURKE, J. (1996). Within-compound associations mediate the retrospective revaluation of causality judgements. Quarterly Journal of Experimental Psychology, 49B, 60-80.

Dickinson, A., ShanKs, D. R., \& Evenden, J. (1984). Judgement of act-outcome contingency: The role of selective attribution. Quarterly Journal of Experimental Psychology, 36A, 29-50.

GLUCK, M., \& BowER, G. H. (1988). From conditioning to category learning: An adaptive network model. Journal of Experimental Psychology: General, 117, 227-247.

KAMIN, L. J. (1968). "Attention-like" processes in classical conditioning. In M. R. Jones (Ed.), Miami symposium on the prediction of behavior: Aversive stimulation (pp. 9-31). Miami: University of Miami Press.

KaO, S. F., \& Wasserman, E. A. (1993). Assessment of an information integration account of contingency judgment with examination of subjective cell importance and method of information presentation. Journal of Experimental Psychology: Learning, Memory, \& Cognition, 19, 1363-1386.

MACKINTOSH, N. J. (1975). A theory of attention: Variations in the associability of stimuli with reinforcement. Psychological Review, 82, 276-298.

MAtute, H., Arcediano, F., \& Miller, R. R. (1996). Test questions modulates cue competition between causes and between effects. Journal of Experimental Psychology: Learning, Memory, \& Cognition, 22, 182-196.

MATUTE, H., VeGas, S., \& De Marez, P. J. (2002). Flexible use of recent information in causal and predictive judgments. Journal of Experimental Psychology: Learning, Memory, \& Cognition, 28, 714-725.

MiLleR, R. R., \& MATUTE, H. (1996). Animal analogues of causal judgment. In D. R. Shanks, K. J. Holyoak, \& D. L. Medin (Eds.), The psychology of learning and motivation: Vol. 34. Causal learning (pp. 133-166). San Diego: Academic Press.
MilleR, R. R., \& MATzEL, L. D. (1988). The comparator hypothesis: A response rule for the expression of associations. In G. H. Bower (Ed.), The psychology of learning and motivation (Vol. 22, pp. 5192). San Diego: Academic Press.

Pavlov, I. P. (1927). Conditioned reflexes (G. V. Anrep, Trans.). London: Clarendon Press.

Pearce, J. M., \& Hall, G. (1980). A model for Pavlovian learning: Variations in the effectiveness of conditioned but not of unconditioned stimuli. Psychological Review, 87, 532-552.

Rescorla, R. A., \& WAGNER, A. R. (1972). A theory of Pavlovian conditioning: Variations in the effectiveness of reinforcement and nonreinforcement. In A. H. Black \& W. F. Prokasy (Eds.), Classical conditioning II: Current research and theory (pp. 64-99). New York: Appleton-Century-Crofts.

SHANKs, D. R. (1985). Forward and backward blocking in human contingency judgment. Quarterly Journal of Experimental Psychology, 37B, 1-21.

ShANKs, D. R., \& Dickinson, A. (1987). Associative accounts of causality judgment. In G. H. Bower (Ed.), The psychology of learning and motivation (Vol. 21, pp. 229-261). San Diego: Academic Press.

Spellman, B. A. (1996). Acting as intuitive scientists: Contingency judgments are made while controlling for alternative potential causes. Psychological Science, 7, 337-342.

Vadillo, M. A., Miller, R. R., \& Matute, H. (2005) Causal and predictive-value judgments, but not predictions, are based on cueoutcome contingency. Learning \& Behavior, 33, 172-183.

Van Hamme, L. J., \& Wasserman, E. A. (1993). Cue competition in causality judgments: The role of manner in information presentation. Bulletin of the Psychonomic Society, 31, 457-460.

Van Hamme, L. J., \& Wasserman, E. A. (1994). Cue competition in causality judgments: The role of nonpresentation of compound stimulus elements. Learning \& Motivation, 25, 127-151.

WAGNER, A. R. (1981). SOP: A model of automatic memory processing in animal behavior. In N. E. Spear \& R. R. Miller (Eds.), Information processing in animals: Memory mechanisms (pp. 5-47). Hillsdale, NJ: Erlbaum.

Wagner, A. R., Logan, F. A., Haberlandt, K., \& Price, T. (1968). Stimulus selection in animal discrimination learning. Journal of Experimental Psychology, 76, 171-180.

WALDMANN, M. R. (1996). Knowledge-based causal induction. In D. R. Shanks, K. J. Holyoak, \& D. L. Medin (Eds.), The psychology of learning and motivation. Vol. 34: Causal learning (pp. 47-88). San Diego: Academic Press.

Waldmann, M. R. (2000). Competition among causes but not effects in predictive and diagnostic learning. Journal of Experimental Psychology: Learning, Memory, \& Cognition, 26, 53-76.

WALDMANN, M. R. (2001). Predictive versus diagnostic causal learning: Evidence from an overshadowing paradigm. Psychonomic Bulletin \& Review, 8, 600-608.

WASSERMAN, E. A. (1990). Detecting response-outcome relations: Toward an understanding of the causal texture of the environment. In G. H. Bower (Ed.), The psychology of learning and motivation (Vol. 26, pp. 27-82). San Diego: Academic Press.

\section{NOTES}

1. The four scenarios used in Experiments 1A and 1B were counterbalanced for order according to the following four different sequences: (1) Scenario 1, Scenario 2, Scenario 3, Scenario 4; (2) Scenario 2, Scenario 1, Scenario 4, Scenario 3; (3) Scenario 3, Scenario 4, Scenario 1, Scenario 2; (4) Scenario 4, Scenario 3, Scenario 2, Scenario 1.

2. The preparation used in Experiment 2 is available at http://www. opineno.com/task.htm.

3. A submaximal intensity of the outcome (i.e., 10/20) was used in Experiment 2 to facilitate the observation of cue competition between A and X relative to Y (see De Houwer et al., 2002, for a rationale) 
APPENDIX

Instructions Used in the Experiments

\section{EXPERIMENTS 1A AND 1B}

\section{First Screen}

In this portion of the experiment, you will be required to learn about various causes and predictors. A cause is something that PRODUCES or brings about an effect, whereas a predictor is something that SIGNALS a future event. Thus, a predictor may or may not also be a cause of the effect. Please read all of the material on each screen carefully before you proceed to the next screen. Periodically, you will be asked to answer several questions, using the mouse to indicate your answer. Simply move the mouse along the rating scale and click on your chosen rating (you can use either the left or the right mouse button to select your rating).

\section{Scenario Screens}

Scenario 1: Naval artillery. Pretend that you are on-board a Naval destroyer in order to observe an artillery demonstration. For this demonstration the gunner is firing shells at a distant target. Each time that the gunner fires a shell, you will observe the shell casing being ejected from the gun and you can also look through your binoculars to observe whether the target has been hit. Other ships are also performing similar demonstrations. Although they are firing at different targets, sometimes their shells will go off course and hit your ship's target. Please try to determine the relationships between the events.

Scenario 2: Auto factory. Pretend that you have recently been hired to work in the quality control division of an auto factory. As part of your job, you are asked to investigate an increase in the rate of defective car bumpers. The car bumpers are manufactured from steel sheets, some of which might be imperfect. As the sheets proceed through the assembly line, a machine designed to detect flaws in the steel sheets sounds an alarm to alert the assembly line workers that an imperfect sheet exists. If the alarm sounds, the workers are supposed to remove the imperfect sheet to prevent a defective bumper from being produced. The workers' supervisor blames the workers for not removing the defective steel sheets when the alarm is sounded, whereas the workers claim that faulty wiring in the bumper stamping machine, which results in power fluctuations, is the real cause of the defective bumpers. Please try to determine the relationships between the events.

Scenario 3: Greenhouse. Pretend that you own a floral supply house that distributes flowers to several local florists. Recently, you hired a new employee to help you in the greenhouse and have been trying to determine if the employee has been doing his job. Each day the employee is supposed to water the flowers for one hour. If the employee waters the flowers as instructed, the flowers should bloom the next day. To water the flowers, the employee turns on a switch that automatically waters them for one hour. Although you are not always present when the employee waters the flowers, you can tell if he has done so by whether or not the floor is wet. However, another employee occasionally fertilizes the flowers, which can also make the flowers bloom the next day. Please try to determine the relationships between the events.

Scenario 4: Strawberries. Pretend that you are a doctor who has been asked to investigate an increase in allergic asthma cases on a produce farm. As you tour the farm, you will see the workers loading crates of fruit onto trucks. You suspect that several of the fruits are responsible for the workers' asthma attacks. As you examine each worker for wheezing symptomatic of asthma, you notice that the shoes of some of the workers are stained from the fruits. Some workers shoes are stained red from strawberries and others are stained blue from blueberries. Please try to determine the relationships between the events.

\section{EXPERIMENT 2}

The National Weather Service (NWS) has opened a number of training positions for research in meteorology. Since you are very interested in enrolling in the NWS (meteorology is your life and their salaries are very appealing), you are going to apply for a position. As a part of this process, you are required to take a computerbased test, which consists of determining the relationships among different events presented on the screen of the computer. Therefore, you have to pay attention to those events so you can then rate their relations ... and obtain a good grade in your exam.

Before taking the exam, it is important for you to remember the difference between causes and predictors. A cause is something that produces or brings about an effect, whereas a predictor is something that only signals a future event.

Remember: You will have to rate the relations among the different events based on your experience with them during the computer-based test.

Good luck! 